(RESEARCH ARTICLE)

\title{
Automated GSM-based un-locker system
}

\author{
Prince Chigozie Iwuji ${ }^{1,}{ }^{*}$ and Julius Achirigbor Idajor ${ }^{2}$ \\ ${ }^{1}$ Electronics and Computer Technology Unit, Department of Physics, University of Calabar, Calabar, Nigeria. \\ ${ }^{2}$ Department of Electrical and Electronic Engineering, University of Calabar, Calabar, Nigeria.
}

Publication history: Received on 09 December 2020; revised on 16 December 2020; accepted on 18 December 2020

Article DOI: https://doi.org/10.30574/wjaets.2020.1.2.0035

\begin{abstract}
This work presents the design and construction of an SMS-based unlocker system that will allow users to lock or unlock a specific door without using the conventional method. The chief component used in the design of this device is the Atmega328 microcontroller (IC1). The device was designed in modules that include the system power supply unit, microcontroller unit, PIR motion sensor, and GSM SIM900 module. These discrete parts or components on their own are made up of smaller components. The different modules were put together in a particular pattern to form the circuit design. SMS from the authorized user's mobile phone enables the Arduino controller to automatically unlock the security door when necessary. Basically, it reads the SMS and acts according to the message command, hence allowing the authorized owner to remotely control the door from anywhere using a mobile phone and preventing unauthorized access to the door. The GSM based door unlocking system constructed worked effectively when tested in homes and office doors and is thus recommended in every home for security purposes.
\end{abstract}

Keywords: GSM-based; Door; Microcontroller; Arduino, Un-locker system

\section{Introduction}

Automation of home appliances with the help of smart home technologies is increasingly getting really common due to the increased comfort, greater safety and security it provides. A home has always been related to a conducive and safe place that provides shelter and protection of assets. Security of household properties has been a major concern for all house owners and their occupants because robbery incidences are increasing day by day as a result of the terrifying and defective security architecture at homes, ventures and businesses [1]. Some technological advancement has been made to secure home appliances from burglars, hoodlums, intruders and thieves. However, most of these high technological securities framework performs effectively with a GSM connection. These technological platforms provide security against planned, regular, human-influenced, accidental and unintended challenges by continuously guarding homes with various tactical means such as smoke, movement, entryway break or glass break sensors [2]. Most home appliances make use of this development in modern technology to improve the quality of life of its occupants. The concept of an automated home has been in existence for a while now and is demonstrated by many actors in innovative movies and novels. However, not many people outside the academic circles will appreciate the fact that automated home technologies are practical and very useful applications that are readily accessible in our everyday lives. Presently, the Global System for Mobile Communication (GSM) technology is effectively deployed in automating our homes and home appliances $[1,3]$.

The GSM-based door un-locker system is a major object of interest that finds its application in different fields, particularly in the area of security. Short message service (SMS) is very common among cellular phone users because it aids in quicker dissemination of information that allows people, networks and devices to share resources, ideas, and other important information, resulting in the speedy transformation of human practice [4, 5]. The GSM based door un-

\footnotetext{
* Corresponding author: Prince Chigozie Iwuji

Electronics and Computer Unit, Department of Physics, University of Calabar, Calabar, Nigeria.
} 
locker system can successfully be constructed using Arduino microcontroller-based devices. This innovation has led to the design and construction of a GSM-based door un-locker system which automatically regulates the servo mechanism to remotely open doors (both for a long and short distance) using SMS. The designed system is a very unique, user's friendly and reliable security apparatus that can be used to address contemporary security challenges at homes. It uses Arduino microcontroller circuitry which is synchronized with the GSM SIM900 module. The switching circuit and the SMS decoder are designed to allow a digital signal processing device to regulate the high power external loads by giving commands encoded as SMS signals. The work is aimed at designing a device that will eliminate the need of being physically present in any location for tasks involving the operation of doors within household/offices, thereby saving time and energy when compared to other security measures. It offers a comfortable, convenient and safe environment for residents and replaced human works with technology. Aside from saving time and energy, the device can be modified to achieve a complete home automation system that will then create a platform for the user to interface between himself and the household. Although, since it is not a real-time control system, the time taken for SMS code to be sent by the controller may vary from a few seconds to a minute depending on the network [6, 7]. The receiver must reside in a location where a signal with sufficient strength can be received from the cellular phone network [8].

\section{Literature}

Ushie, Donatus \& Akaiso (2013) designed and constructed a prototype security door that can be controlled remotely using a GSM acting as the transmitter and another GSM having a dual-tone multi-frequency (DTMF) connected to the security door's motor using a dual-tone multi-frequency decoder linked to a microcontroller unit and a stepper motor. The designed prototype consists of four major modules, namely; the decoding module, the GSM module, the switching module, and the controlling module. The GSM module acts as both the transmitting and receiving units. It uses a smartphone to establish a link between the user at one end and the door at the receiving end. The controlling module and the decoding module operates using a modern integrated circuit chip to ensure appropriate conversion of the signal from its analog form to binary or digital codes. This enables the microcontroller to properly communicate with the switching device responsible for closing and opening the door. The codes used for the designed project was written using assembly language along with visual basic software and M-IDE studio for MC-51 compiler. The designed security device worked perfectly with the window XP environment without error before being burnt into the microcontroller sockets to its equivalent PIN.

The design and implementation of an electronic door lock/unlock system using the Arduino was carried out by Umbarkar, Rajput, Halder, Harname \& Mendgudle in 2016. This security system locks or unlocks the door using three different methods that include Keypad, Bluetooth, and GSM modules. A blue-tooth application from a smartphone can be used to open or close the door. Also, the keypad or a four-digit message from a GSM device can as well be used to open or close it. If an unauthorized person tries to enter the password in any of the systems three unsuccessful times consecutively, then the Arduino controller will send a message to the owner and also initiating the warning alarm against the unauthorized user.

In 2018, Usha, Rajkumar, Shyamala, Saranya worked on GSM based door open and closed device. The device's control unit sends a command to the mobile user at the receiving end. The command that is based on the GPRS and GSM service is used to lock or unlock the door. The command sent from the control unit to any authorized person consists of the person's username and the password that will be used for the door operations. If there is an unauthorized person(s) on the door, the device's IR sensor will signal the intensity breakout that triggers the alarm. The device has several security applications in places such as homes, shops, offices, and also in industries. It replaces the conventional security system which depends on the use of a key to open or close a door. The device designed by them needs no physical key to operate the door. Any authorized user operates the required door using an SMS as a key.

Some of the works carried out are based on DTMF technology that makes it prone to errors. Latency is another challenge of the DTMF based security device. The process of filtering the frequency range causes latency. Power consumption is high since it takes a longer time to process information or code through DTMF based security apparatus than SMS based Arduino microcontroller device. Due to improvement in technology and the need to design devices in which power consumption is low and less prone to error; a very reliable, portable, fast, simple, and compatible with all networks device were designed and constructed. 


\section{Material and methods}

\subsection{Materials}

The material used in the construction of this security device can be easily sourced for except the sim900 modem and Atmega328 circuit. Each of the materials used for the design has its specific and unique functions. Some of the components used in the design are microcontroller, Arduino Uno Hardware (Data processing unit), transistors, resistors, capacitors, conductors, sim900 modules, DC motors, etc.

\subsection{Methodology}

The circuit complexity was significantly reduced by the use of a PIC microcontroller. This was done to ensure it fits into modern technology that is mostly miniaturized, cost-effective, and easy to use. A modular approach was adopted in this design. There are four different modules that combine to form the full work. These discrete parts or components on their own are made up of smaller components. The modules are combined in a definite pattern to form the overall circuit design. The various modules required for the operation of the central system and their operations are shown in figure 1 below.

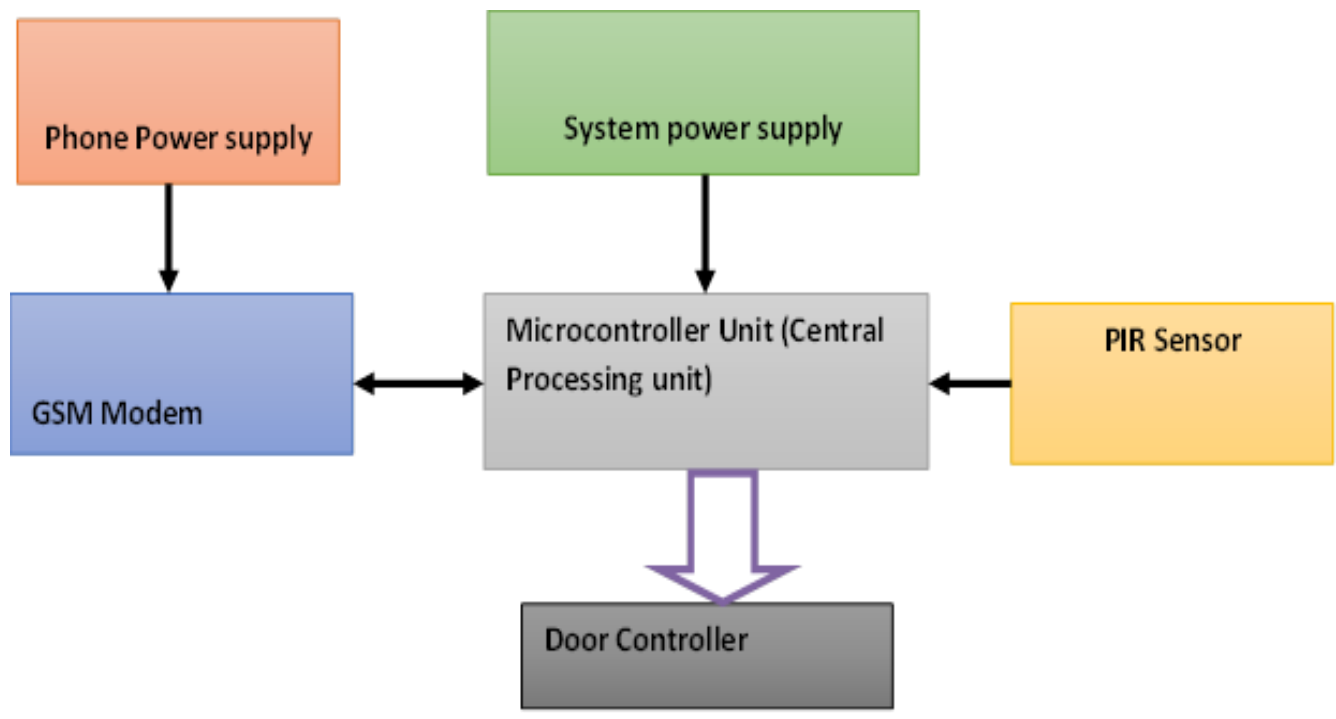

Figure 1 Block diagram of the GSM based door un-locker system

The in-built power supply consists of a regulated 5V DC which is used to power the PIR sensor and its internal circuitry. It also has an unregulated $12 \mathrm{~V} \mathrm{DC}$ which is used to power the microcontroller. The main task in designing this smart based door un-locker system that is capable of monitoring and controlling movement in and out of a door is the development of the central control system that controls the notifying alarm system. The device processes the information within a short time. The control circuitry was designed to solve the problem of controlling and allocating different tasks. For this reason, the control circuitry was designed to have a fast operating speed, several input/output ports, large memory capacity, and low power consumption. The control board voltage regulator that houses REG1 is an LM317T 1.5A adjustable voltage regulator that provides a nominal $1.25 \mathrm{~V}$ between its OUT and adjustable (ADJ) terminals. The output voltage from the regulator is set to function by the $120 \Omega$ resistor and series $470 \Omega$ resistor between the OUT and ADJ terminals. Using the $470 \Omega$ resistor and assuming the constant reference voltage to be $1.25 \mathrm{~V}$, then the output voltage is derived using the formula as shown below.

$$
\begin{gathered}
V_{\text {out }}=V_{\text {ref }}\left(1+\frac{R_{2}}{R_{1}}\right) \\
V_{\text {out }}=1.25\left(1+\frac{470}{120}\right) \\
V_{\text {out }}=6.14 \mathrm{~V}
\end{gathered}
$$

To obtain the series resistor for status LED, we use ohm's law. 
From ohm's law:

$$
R=\frac{V}{I}
$$

The calculation can be reduced to a single formula:

$$
R=\frac{V_{S}-V_{D}}{I}
$$

Where $\mathrm{R}=$ resistor, $\mathrm{V}_{\mathrm{S}}=$ supply voltage, $\mathrm{V}_{\mathrm{D}}=\mathrm{LED}$ voltage drop and $\mathrm{I}_{\mathrm{LED}}=$ current through LED.

$$
R=\frac{V_{S}-V_{D}}{I_{L E D}}
$$

$R=\frac{5-2}{0.02}=350 \Omega$

The nearest conventional value for $R$ available is $350 \Omega$.

\section{Design, construction and implementation of the automated door unlocker device}

The design, construction, and implementation of the device hardware were done using the following procedures. Firstly, the schematic and layout diagram was designed followed by mounting and soldering the different components on the printed circuit board (PCB). The final stage was the casing of the device. The circuit was first developed using a computer program known as Proteus. This program help in the simulation of the project before it is physically implemented. Simulation of the circuit using Proteus makes it easier to ascertain and make corrections and decisions before final implementation. As design criteria, the design of the schematic and printed circuit board is an important process in the accomplishment of this project. The production of an effective and reliable PCB creates better performance and more consistent results. Crosschecking of footprints and design procedures was necessary to ensure a favored outcome. After designing the schematic diagram, the next step was to convert it into a printed circuit board (PCB). The circuit diagram is shown below in figure 2 .

The chief component used in the design of this device is the Atmega328 microcontroller (IC 1 ). A few other external components interface with the Atmega328 microcontroller. The power unit of the circuit is made up of a $5 \mathrm{~V}$ voltage regulator that maintains a constant $+5 \mathrm{~V}$ DC supply to the Atmega328 microcontroller, the PIR sensor, and the GSM modem. The ATMega328 includes 8192 bytes of code (FLASH) memory, 512 bytes of RAM, and 512 bytes of data (EEPROM) memory. This microcontroller comes with four 8-bit input/output (I/O) ports. Ports " $\mathrm{A}$ " and " $\mathrm{C}$ " are used for the external interface, while Port "B" is used to drives the five status LEDs (LED 1 - LED $)$ and is also used for in-system programming (ISP) via CON5. The upper three bits of Port PD - PD7 i.e, Port D are used to read the state of jumpers JPJP3. The lower two bits $\left(\mathrm{PD}_{0} \& \mathrm{PD}_{1}\right)$ are programmed as serial transmit and receive lines for communication with the GSM modem. Power for the microcontroller and its associated circuitry is provided by a $7805+5 \mathrm{~V}$ regulator (REG 1 ). A reverse-polarity D1 is used to protect the input of the regulator. The two outputs of the microcontroller are provided for controlling external devices and the electric motor used in the circuit design. Each output line is driven by one opencollector transistor pair type BC547. One of the circuit input ports is the digital input Port Pin PB1 which is made available for sensing the state of the external triggering device (PIR Sensor). The microcontroller samples these inputs to see if there is any signal from the PIR sensor. 
World Journal of Advanced Engineering Technology and Sciences, 2020, 01(02), 044-051

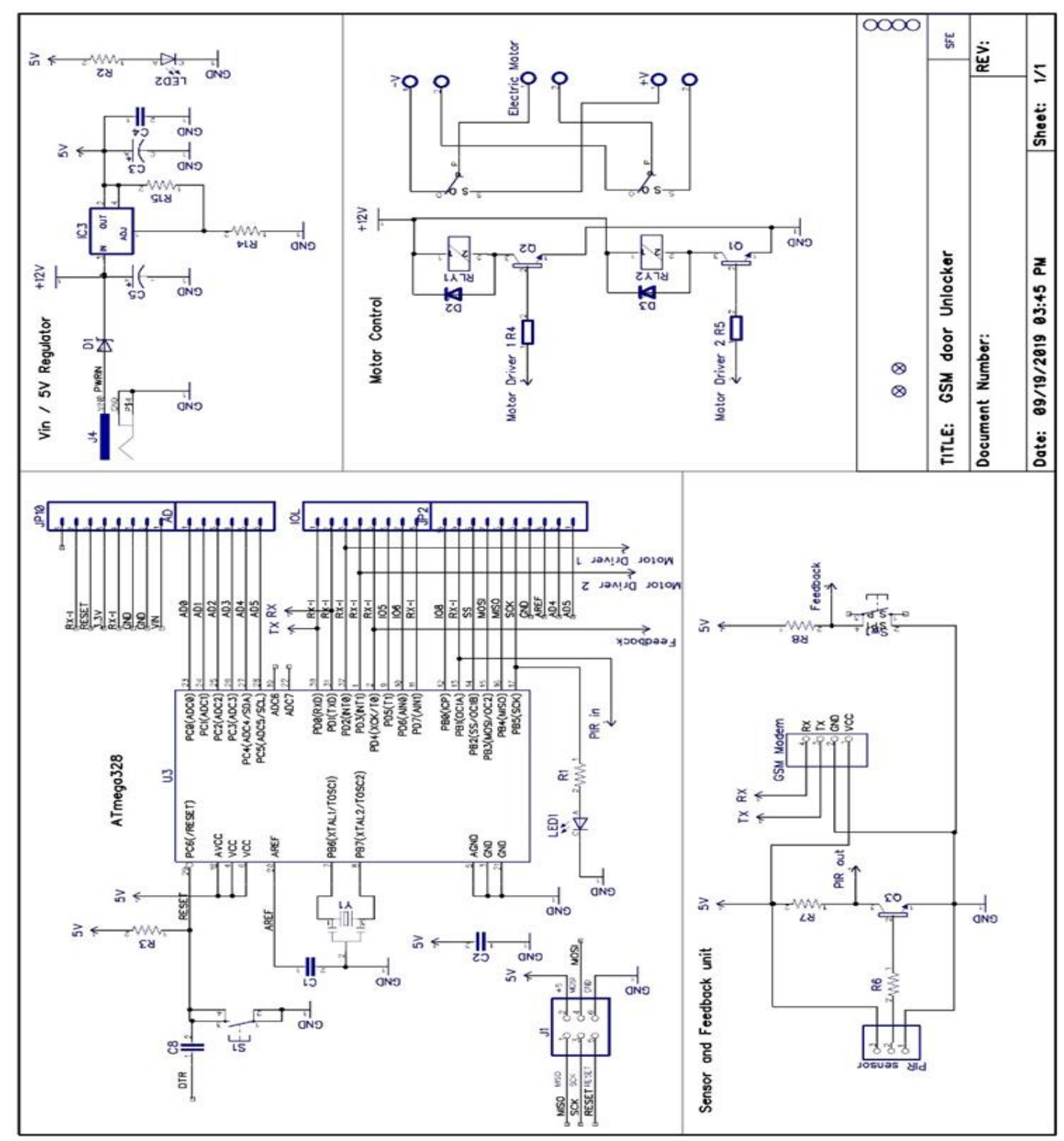

Figure 2 Complete circuit diagram for the GSM Door Unlock system

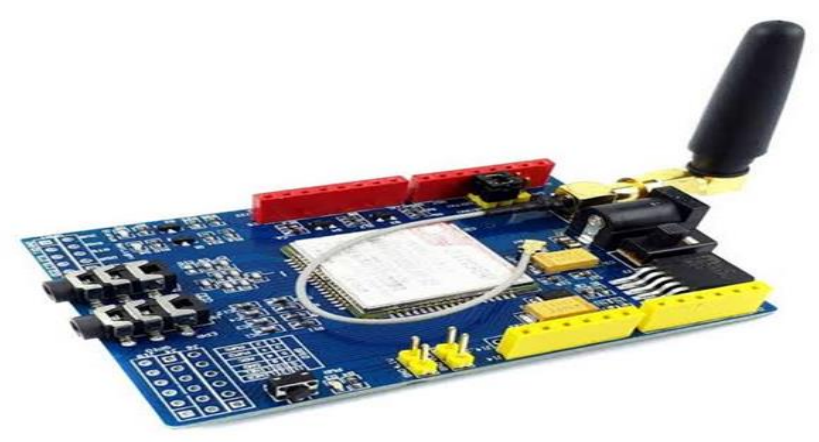

Figure 3 Diagram of the GSM module 


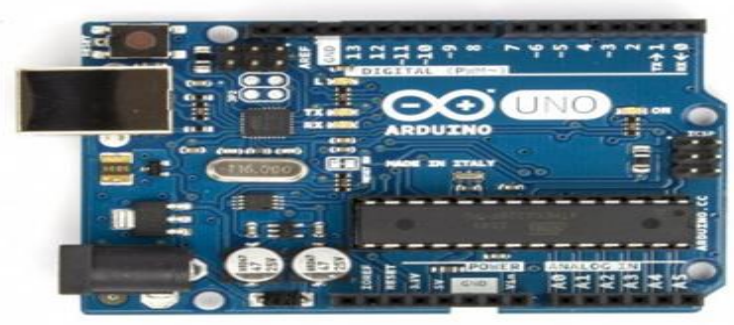

Figure 4 Diagram of the arduino uno board.

In mounting and soldering of components on the printed circuit board, a check on the printed circuit (PC) board for short circuit tracks or break of the copper was carried out first. A check on the hole sizes was also carried out to ensure it fits the different components. Populating the printed circuit board shown below did not present any problem because the board is spacious and only lead components were used. Figure 5 below shows the printed circuit board layout for the main control board.

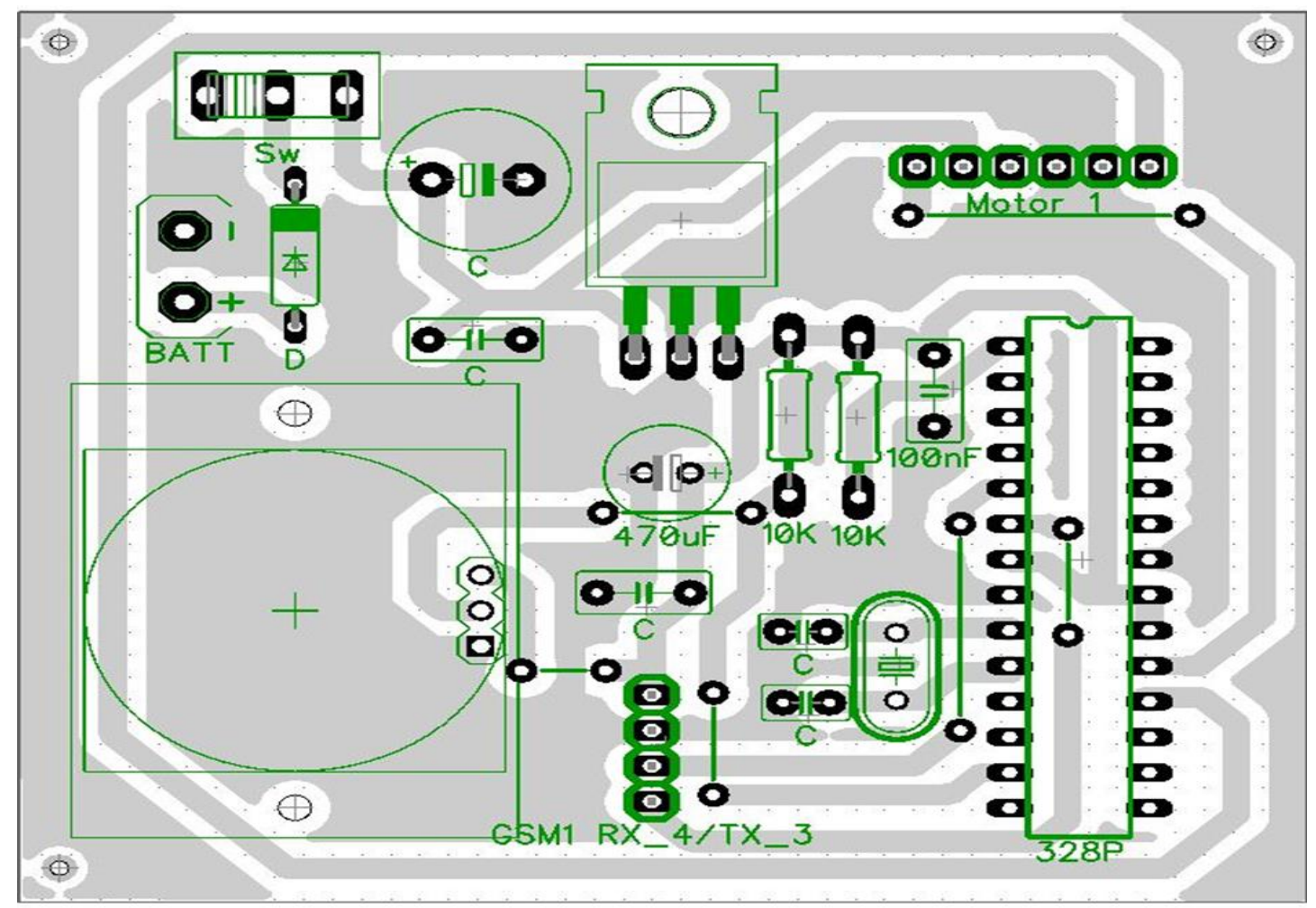

Figure 5 Printed circuit board layout for the main control board

Care was taken to ensure that the labeling and the components on the printed circuit board were taken into consideration. After checking the tracks and the size of the holes, component assembly followed with the installation of the link wires and resistors. Before mounting the resistors, a digital Multi-meter was used in checking each resistor value before soldering it onto the printed circuit board. After this stage, Capacitors were installed, with care taken to ensure that the electrolytic capacitors are oriented correctly.

\section{Testing and casing of the device}

Before mounting the components on the PCB, the individual components were tested to ascertain their workability. The testing of the device was carefully carried out to check that all components were properly connected and oriented correctly. A digital multimeter was used to test voltage levels at each point in the circuit. This was to ensure that no excessive voltage gets into the circuit. Testing between the ground and output of the supply board confirms that $+5 \mathrm{v}$ and +7.2V DC were present. This is the value delivers by the DC battery to the voltage regulator and the microcontroller 
circuitry. After the basic test, the device was connected to the mains power source and the sensor kept away from moving person or object. This was done to prevent false triggering of the device. The device was then installed on the door in such a way that the sensor was positioned to optimally track intruders and authorized users. After the entire test, the device was confirmed to function properly on the door according to specifications.

The casing is very important for the protection of the device to ensure longevity. After the construction and testing of the device, it was neatly and carefully housed in a casing made of a synthetic plastic material called 'Perspex'. Perspex was chosen because of its durability, safety, and remarkable dark background. A drilling machine was used to create a point for the connector of the battery terminal and other fittings. The necessary considerations made when forming the casing were the material used for its construction, physical size, and appearance. These considerations define durability, physical appearance, and corrosion resistance. Based on the above consideration, Plastic Perspex casing was used. The Perspex piece was shaped into an appropriate size. The pieces were then glued together using special glue called " $U H U$ ALLPLAST" to form the rectangular box casing used. The casing was done in such a way that it can be opened by screwing the nuts on the top of the casing to get access to the main circuit in case of maintenance and repair. Double-checking and testing were done to ensure that the device functions very well before closing the casing.

\section{Operation of the device}

The device is installed in the door and the sensor is kept in a safe place closed to the door. When the digital inputs of the security system change state, the controller sends a pre-programmed SMS message to the designated mobile number. The message is used in opening and closing the door when it senses the presence of a person within the coverage area of the PIR sensor. The in-built command known as the number of unique messages is also recognized by the controller.
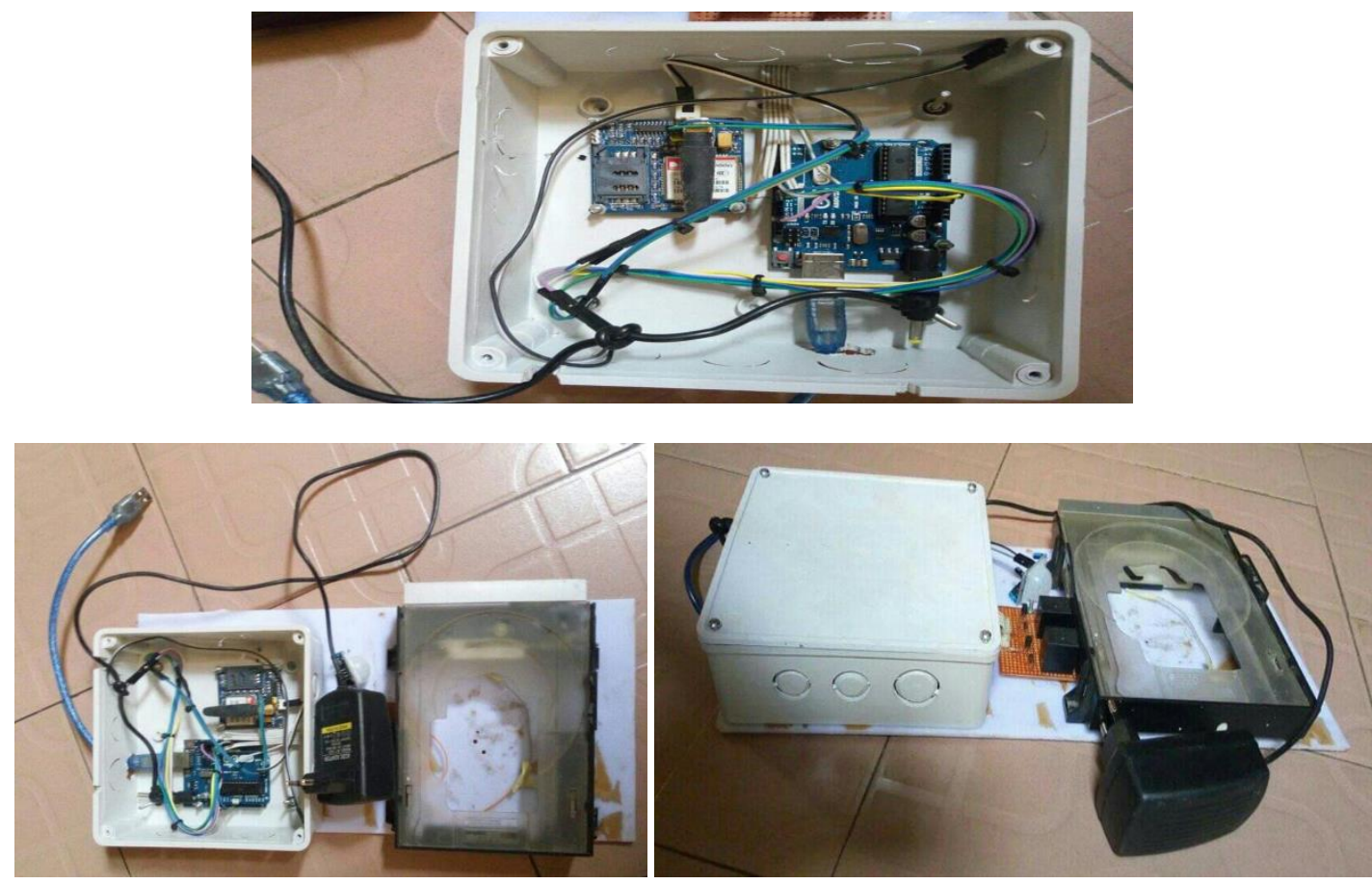

Figure 6 a, b \& c Picture of the constructed GSM based door unlocker System

\section{Conclusion}

GSM based door un-locker System was designed and constructed. A Modular approach was adopted in this design. There are four different modules that combine to form the full work. These discrete parts or components on their own are made up of smaller components. The modules are combined in a definite pattern to form the overall circuit design. The chief component used in the design of this device is the Atmega328 microcontroller (IC1). The system utilizes user input as a key for users' access through the use of short message service SMS. It has been successfully demonstrated and will 
serve as a device for securing personal wares in environments where it is deployed against unauthorized users by setting off appropriate configuration for every action.

\section{Recommendation}

It is recommended from this work that further research be carried out in other to design and construct a hybrid model that will integrate SMS, face detection and fingerprint to lock or unlock security doors.

\section{Compliance with ethical standards}

\section{Acknowledgments}

We wish to sincerely thank the staff of the Department of Physics and the Department of Electrical and Electronic Engineering for their advice and encouragement during the course of writing this article. Our honest appreciation also goes to Tom Elijah for his contributions to the success of this article.

\section{Disclosure of conflict of interest}

The authors of this article declare that there is no conflict of interest be it financial, professional, personal, knowledge, affiliation or otherwise.

\section{References}

[1] Alkar AZ, Bushur U. An Internet Base Wireless Home Automation System for Multifunctional Devices. IEEE Transaction on consumer Electronic. 2015; 51(2): 1169-1174.

[2] Usha V, Rajkumar N, Shyamala DM, Saranya JC. GSM based door open and closing system. International Journal of Engineering \& Technology. 2018; 7 (2.4): 97-100.

[3] Umbarkar S. Rajput G, Halder S, Harname P, Mendgudle S. Keypad/Bluetooth/GSM Based Digital Door Lock Security System. Advances in Intelligent Systems Research. 2016; 749-757.

[4] Ushie JO, Bassey DE, Akaiso E. Design and construction of door security system using GSM. International Journal for Engineering and Computer Science. 2013; 2(3): 2235 - 2257.

[5] Parab SA, Joglekar A. Implementation of home security system using GSM module and microcontroller. International Journal of Computer Science and Information Technologies. 2015; 6(3): 2950 - 2953.

[6] Hassan R, Khan MM, Ashek A. Design and implementation of a microcontroller based home security system with GSM technology. Open Journal of Safety Science and Technology. 2015; 5(6): 55 - 62.

[7] Bangali J, Shaligram A. Design and implementation of security system for smart home based GSM technology. Internation Journal of Smart Home. 2013; 7(2): 201 - 206.

[8] Aggarwal A, Joshi RC. WSN and GSM based remote home security system. International Conference on Recent Advances and Future Trends in Information Technology Proceedings published in International Journal of Computer Applications. 2013. 